\title{
O Desempenho de Adultos Jovens e Idosos na Iowa Gambling Task (IGT): Um Estudo sobre a Tomada de Decisão
}

\author{
Decision-making Capacity of Young Adults and Older Adults as Measured by \\ the Iowa Gambling Task (IGT)
}

\author{
Daniela Di Giorgio Schneider*, Maria Alice de Mattos Pimenta Parente \\ Universidade Federal do Rio Grande do Sul, Porto Alegre, Brasil
}

\begin{abstract}
Resumo
O presente estudo teve por objetivo investigar a tomada de decisão em jovens e idosos. O instrumento utilizado foi a Iowa Gambling Task, a qual foi adaptada para o Português Brasileiro no presente estudo. Participaram do estudo 82 indivíduos, 42 jovens de 20 a 35 anos e 40 idosos de 61 a 78 anos. Os resultados demonstraram que não houve diferença entre os grupos quanto às escolhas de cartas de cada baralho. Ambos os grupos selecionaram mais cartas dos baralhos "A" e "C" e menos cartas dos baralhos "B" e "D". Não houve diferença entre os grupos, no que se refere ao aprendizado desenvolvido ao longo da tarefa. Jovens e idosos foram gradualmente adquirindo uma predileção pelos baralhos "C" e "D”, a qual culminou no quarto bloco, porém não se manteve no último. Peculiaridades na aplicação do instrumento podem responder pela não manutenção do aprendizado.

Palavras-chave: Tomada de decisão; envelhecimento; busca e aversão ao risco.
\end{abstract}

\begin{abstract}
The study aimed to analyze the decision-making capacity of young adults and older adults. The Iowa Gambling Task, which was adapted for the Brazilian Portuguese in the present study, was used to measure decisionmaking. The sample consisted of 82 healthy persons divided in two groups: Group $1-42$ young adults and Group 2 - 40 elders. The results showed older adults no differences between the groups regarding the choices in the cards. Both groups showed a preference for decks "B" and "D". Regarding the learning process acquired during the test, there were also no differences between the two groups. All the subjects gradually developed a predilection for the cards of decks "C" and "D", preference that culminated in the fourth block, although not sustained up to the last one. This lack of maintenance of the learning process could perhaps be explained by some particular existing peculiarities in the application of the instrument.

Keywords: Decision making; aging; risk-taking; risk-avoiding.
\end{abstract}

A tomada de decisão (TD) pode ser considerada uma função cognitiva fundamental para uma satisfatória interação do indivíduo com seu contexto social. Diariamente as pessoas são requeridas a decidirem entre diversos cursos de ação, onde nem sempre a opção mais favorável encontra-se evidente. Isto exige do ser humano, não somente perspicácia na hora de solucionar tais dilemas cotidianos, como também flexibilidade ao considerar cada situação individualmente, suas características e consequiências, em um tempo presente e futuro (Palmini, 2004).

Dentro do paradigma cognitivo, a TD é definida como uma função complexa que envolve a escolha entre duas ou mais opções, demandando a análise das características dessas opções e a estimativa de conseqüências futuras acarretadas pela escolha (Ballesteros Jiminez \& Garcia Rodríguez, 1996; Eysenck \& Keane, 1994; Medin \& Ross, 1992; Plous, 1993; Tversky \& Kahneman, 1974). No entender de Antonio

\footnotetext{
* Endereço para correspondência: Rua Santa Cecília, 1556, Porto Alegre, RS, 90420-040. Fone: (51) 3332-3249; Fax: (51) 3331-6403. E-mail: danigiorgio@hotmail.com

Este trabalho contou com o apoio da CAPES
}

Damasio (1996), o processo de decisão supõe que o indivíduo conheça: (a) a situação que requer determinada decisão, (b) as distintas possibilidades de ação, e (c) as conseqüências imediatas e futuras de cada uma destas ações.

Uma atenção especial vem sendo dada a esta função cognitiva desde a década de 70, quando Daniel Kahneman e Amos Tversky publicaram seus achados em julgamento e TD (Kahneman \& Smith, 2002; Kahneman \& Tversky, 1979; Mellers, Schwartz \& Cooke, 1998; Tversky \& Kahneman, 1974). Apesar de grandes estudos estarem sendo conduzidos desde então, dando origem a diversos modelos teóricos, a influência da emoção e seus substratos neurológicos nos processos decisionais tem sido extensamente negligenciada. Somente a partir da década de 90, devido, em parte, aos trabalhos de Damasio, Bechara et al., os processos biológicos subjacentes à TD também têm sido considerados. Nestes é salientado o papel da emoção e seus substratos neurológicos na TD e na cognição de pacientes com lesões cerebrais focais. O córtex pré-frontal tem sido identificado como responsável por subservir funções integrativas chaves, entre elas, atenção, controle executivo, planejamento, memória de trabalho, conteúdo emocional e motivacional e TD 
(Bechara, 2001, 2003; Bechara, Damasio, Damasio \& Anderson, 1994; Bechara, Damasio, Tranel \& Damasio, 1997; Bechara, Tranel \& Damasio, 200ob; Bechara, Tranel, Damasio \& Damasio, 1996; A. Damasio, 1996).

António Damasio (1996) desenvolveu um modelo teórico, a Hipótese do Marcador Somático, o qual vem embasando estudo dos substratos neurológicas da TD. Segundo concepções deste modelo, sinais emocionais atribuem valor a determinadas opções e cenários, funcionando como tendências ocultas ou evidentes, que induzem à decisão. Diante de uma situação de decisão, antes de aplicar qualquer análise de custo e benefício às situações e raciocinar visando à resolução do problema, o indivíduo depara-se com uma sensação corporal automática, que são os marcadores somáticos. Por exemplo, quando surge em sua mente um mau resultado associado a uma dada opção de resposta, alguma sensação corporal (somática) desagradável ocorre. Neste momento, o marcador somático direciona a atenção para o resultado negativo a que a ação pode conduzir e atua como um sinal de alarme automático. A partir deste alerta, é possível que o sujeito rejeite imediatamente o rumo de ação negativo e tenha que escolher entre um número muito menor de alternativas. Este processo conta com a ajuda de componentes de aprendizagem (através da associação entre categorias específicas de estímulos e categorias específicas de estados somáticos), da capacidade atencional e da memória de trabalho, para que seja possível sua ocorrência (Bechara, Damasio, Tranel \& Andeson, 1998; A. Damasio, 1996, 2003; Fellows \& Farah, 2003a).

Segundo António Damasio (2003) a TD é processada a partir de duas rotas complementares: a rota "A" que pressupõe ponderar opções para ação e antecipar resultados futuros através de estratégias de raciocínio; e a rota "B" que, em paralelo, induz a ativação de experiências emocionais anteriores em situações semelhantes (Figura 1). Neste último, a recordação de material emocionalmente relacionado, seja manifesto ou oculto, influencia o processo de TD focando a atenção na representação de resultados futuros ou interferindo nas estratégias de raciocínio. Às vezes, o caminho B pode levar diretamente à decisão, como por exemplo, quando uma sensação somática incita uma resposta imediata. Desta forma, este modelo pressupõe uma clara integração entre os processos cognitivos e os processos emocionais.

Os estudos que focalizaram o efeito de idade em tarefas de tomada de decisão ainda apresentam dados contraditórios. Walker, Fain, Fisk e McGuire (1997) não observaram efeito da idade na velocidade e na qualidade da tomada de decisão, em uma tarefa que incluía habilidades relacionadas ao ato de dirigir. A ausência de diferenças também foi observada no estudo de Hershey e Wilson (1997) sobre a qualidade das decisões acerca da designação financeira em jovens e idosos. Por outro lado, em tarefas que exigem maior grau de julgamento e avaliação, os piores desempenhos de idosos apontam uma gradual perda da flexibilidade cognitiva (Chasseigne, Mullet \& Stewart, 1997, citados por Sanfey \& Hastie, 2001; Finuncane et al., 2002).

Trabalhos utilizando a Iowa Gambling Task(IGT), instrumento desenvolvido de acordo com a teoria do marcador somático e utilizado na clínica neuropsicológica, também mostram resultados contraditórios quanto ao efeito de idade. Bechara et al. (1994) e MacPherson, Phillips e Sala (2002) não encontraram diferenças entre jovens e idosos. Entretanto, mais recentemente, Denburg, Tranel e Bechara (2005) observaram que idosos exibiriam uma TD prejudicada quando comparados aos jovens.

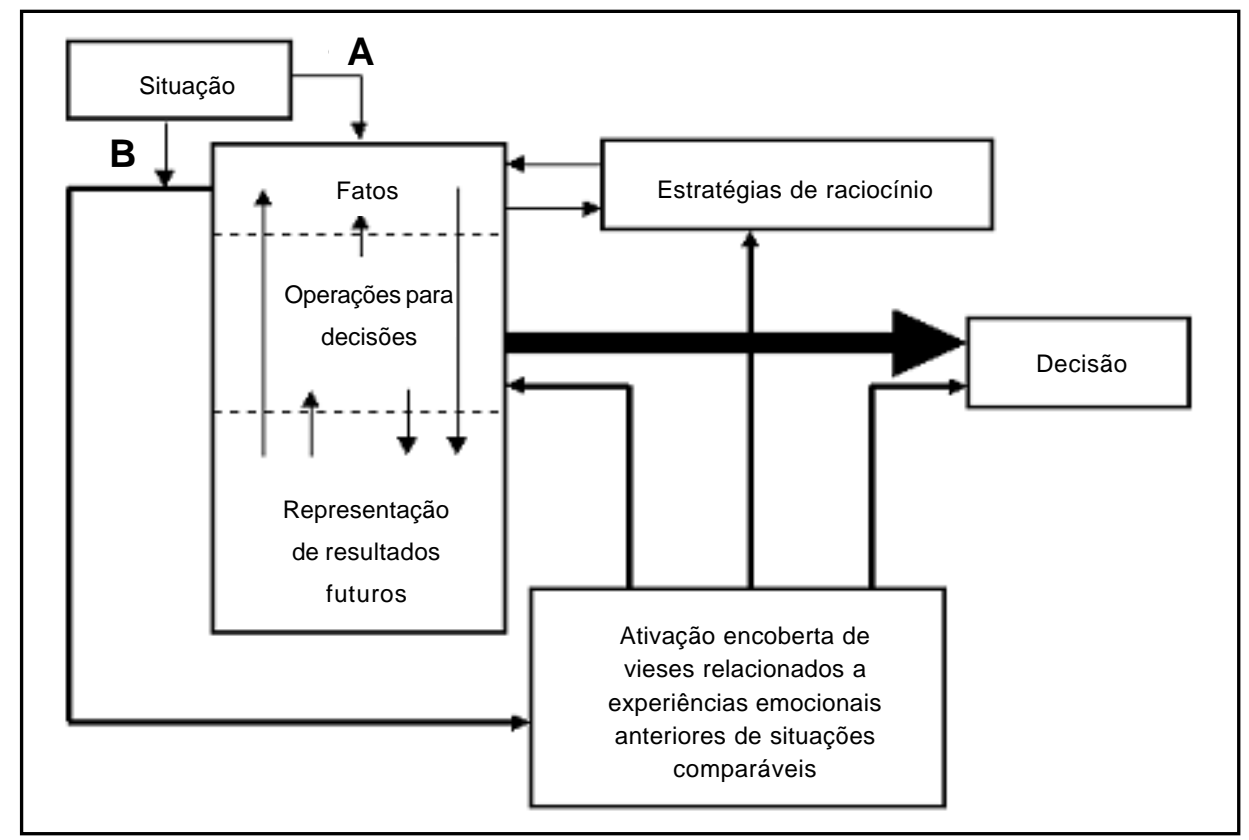

Figura 1. Esquema do modelo do Processo de Tomada de Decisão, com base na Hipótese do Marcador Somático (Bechara, 2003). 
Por fim, Dror, Katona, e Mungur (1998) e Sanfey e Hastie (2001) demonstraram que tanto idosos como jovens têm igual capacidade cognitiva de decidir em situações de risco, com diferenças qualitativas em termos comportamentais de busca ou aversão ao risco, argumentando que adultos mais velhos consideram menos informações antes de executarem uma escolha devido às falhas na capacidade da memória de trabalho.

O presente estudo pretendeu contribuir com o debate a respeito das diferenças entre idade na tomada de decisão, especificamente no que tange ao comportamento de busca ou aversão ao risco, a partir do seu desempenho na IGT (Bechara et al., 1994). Os objetivos específicos do presente estudo incluíram: (a) investigar a influência dos baralhos no comportamento de decidir de ambos os grupos, tendo em vista as diferentes características dos quatro baralhos que compõem a IGT (Bechara et al., 1994) que se diferem quanto à possibilidade ou não de ganhos em longo prazo e quanto à freqüência de perdas; (b) averiguar se houve diferença no aprendizado desenvolvido por ambos os grupos, ao longo da tarefa; e, (c) adaptar a IGT para o Português Brasileiro.

\section{Método}

\section{Participantes}

A amostra constituiu-se de 82 indivíduos de ambos os sexos, da população de Porto Alegre e região metropolitana, distribuídos em dois grupos de acordo com sua faixa etária. O grupo de jovens englobou 42 indivíduos, com idades entre 20 e 35 anos (média de idade 24 anos, desvio padrão 4,43) e o grupo de idosos foi composto por 40 indivíduos com idade entre 61 e 78 anos (média de idade 68 anos, desvio padrão 5,01). A seleção dos participantes foi realizada a partir da técnica de amostragem não-aleatória de conveniência. Os idosos foram selecionados em centros de atividades sociais voltados para terceira idade e os jovens foram recrutados, na sua maioria, em ambientes universitários. Foram excluídos da amostra aqueles participantes que apresentavam algum transtorno psicológico, declínio cognitivo leve, processos demenciais, distúrbios de visão e audição e familiaridade aos testes utilizados ou envolvimento freqüente em investimentos financeiros. A escolaridade mínima dos participantes aceita para este estudo, foi igual ou superior a oito anos completos, o que equivale à conclusão do Ensino Fundamental. A Tabela 1 apresenta a caracterização da amostra segundo gênero e nível de escolaridade, distribuída por grupo.

\section{Instrumentos e Procedimentos}

Os instrumentos apresentados a seguir foram administrados individualmente, em uma única vez, no Laboratório de Neuropsicolinguística da Universidade Federal do Rio Grande do Sul ou na residência do próprio participante. Primeiramente foram aplicados os instrumentos de triagem, para, posteriormente, ser administrada a IGT. O Mini Exame do Estado Mental (MEEM) e os Critérios de Positividade para Déficit Cognitivo foram aplicados apenas no grupo de idosos, tendo em vista que os indivíduos do grupo de jovens eram, em sua maioria, estudantes universitários que mantinham atividades intelectuais constantes. Todos foram conduzidos manualmente, sendo a IGT administrada através do computador. A participação dos indivíduos neste estudo foi voluntária, tendo todos os participantes assinado o consentimento livre e esclarecido. O projeto foi apresentado à Comissão de Ética do IPUFRGS.

\section{Questionário sócio-cultural}

Este questionário foi desenvolvido através de uma adaptação do questionário utilizado pelo Laboratório de Neuropsicolinguística da UFRGS, e teve como objetivo coletar dados e de identificação da amostra e informações sócio-culturais.

Tabela 1

Caracterização da Amostra quanto ao Gênero e Escolaridade, Idade e Escores no MEEM, conforme os Grupos

\begin{tabular}{|c|c|c|c|}
\hline \multicolumn{4}{|c|}{ Grupos } \\
\hline Sexo & Jovens n (\%) & Idosos n (\%) & Total \\
\hline Masculino & $14(33,3)$ & $13(32,5)$ & $27(32,9)$ \\
\hline Feminino & $28(66,7)$ & $27(67,5)$ & $55(67,1)$ \\
\hline \multicolumn{4}{|l|}{ Escolaridade } \\
\hline A té $2^{\circ}$ grau incompleto & $\mathrm{O}(0)$ & $10(25,0)$ & $10(12,2)$ \\
\hline \multicolumn{4}{|l|}{ De $2^{\circ}$ grau completo a } \\
\hline $3^{\circ}$ grau incompleto & $17(40,5)$ & $13(32,5)$ & $30(36,6)$ \\
\hline \multirow[t]{2}{*}{$3^{\circ}$ grau completo } & $25(59,5)$ & $17(42,5)$ & $42(51,2)$ \\
\hline & Jovens m (dp) & Idosos m (dp) & \\
\hline Idade & $24(4,43)$ & $68(5,01)$ & \\
\hline MEEM & & $27,3(1,26)$ & \\
\hline Total $(\mathrm{n})$ & 42 & 40 & 82 \\
\hline
\end{tabular}




\section{Mini Exame do Estado Mental (MEEM)}

O presente instrumento, adaptado para o Português Basileiro por Folstein, Robins e Helzer (1983) e para a população de Porto Alegre por Chaves e Izquierdo (1992), foi empregado neste estudo no grupo de idosos, com o intento de excluir indivíduos que apresentem critérios positivos para demência ou distúrbios neurológicos. Avalia cinco áreas cognitivas: orientação temporo-espacial, capacidade de decodificação verbal, cálculo e atenção, memória de curto prazo e linguagem. Foram excluídos idosos que não atingiram 24 pontos neste instrumento.

\section{Critérios de positividade para déficit cognitivo}

Utilizou-se a escala de "Critérios de Positividade para Déficit Cognitivo”, adaptada por Ferreira (1997), com o fim de excluir os idosos portadores de Declínio Cognitivo Leve. Os critérios diagnósticos para este distúrbio incluem: queixa de memória, atividades diárias normais, funções cognitivas gerais normais, função da memória anormal para a idade e ausência de demência (Petersen et al., 1997). Foram excluídos da amostra aqueles participantes idosos que não atingiram a pontuação exigida para, ao menos, um subteste. Aplicou-se uma versão resumida da escala, na qual foi investigada a memória dos participantes, a partir das seguintes testes: seqüência de dígitos, lista de palavras, reconhecimento de faces, memória viso-espacial, memória imediata e memória recente.

\section{Breve Entrevista Internacional de Neuropsiquiatria Modificada (M.I.N.I.)}

O M.I.N.I (Sheehan et al., 1998) foi aplicado no presente estudo com o intuito de retirar da amostra portadores de transtornos psiquiátricos. Consta de uma breve entrevista estruturada para a triagem dos principais quadros psicopatológicos, validada para a População Brasileira por Amorim (2000). Seus critérios diagnósticos são semelhantes àqueles encontrados no DSM-IV TR (Manual Diagnóstico e Estatístico dos Transtornos Mentais) e no CID-10 (Código Internacional de Doenças, $10^{\mathrm{a}}$ edição). Foram excluídos os participantes que atenderam aos critérios para algum dos transtornos psiquiátricos avaliados.

\section{Iowa Gambling Task (IGT)}

Para avaliar a TD foi utilizada uma versão adaptada computadorizada da IGT, elaborada originalmente por Bechara et al. (1994). Esse instrumento contempla uma situação de tomada de decisão sob incerteza, que envolve escolhas monetárias, permitindo classificar o comportamento de decisão do indivíduo em termos de aversão ou busca pelo risco. A tarefa envolve escolhas de uma carta, ao longo de 100 jogadas (cinco blocos de vinte jogadas cada), de um dentre quatro baralhos. Cada um desses trabalhos inclui uma longa série de ganhos e perdas. A partir de um processo de aprendizagem, os participantes criam padrões de probabilidade e inferem quais baralhos são vantajosos e quais não o são. Esses devem desenvolver o conhecimento de quais baralhos são arriscados e quais são lucrativos em longo prazo. Cada baralho possui um esquema fixo e pré-programado de recompensa e punição. Escolhendo qualquer carta do baralho "A" ou do "B", ocorre um rendimento de $\mathrm{R} \$$ 100,00 ; já optando por qualquer carta dos baralhos "C" ou “D” o rendimento é de $\mathrm{R} \$ 50,00$. No entanto, o lucro futuro final de cada baralho varia, porque a punição é, em termos de valor, maior nos baralhos que pagam altas quantias (A e B) e menor nos baralhos que pagam menos (C e D). Por exemplo, depois de virar dez cartas do baralho "A", os participantes ganham $\mathrm{R} \$ 1.000,00$, mas também se deparam com cinco punições não previstas $(\mathrm{R} \$ 150,00 ; \mathrm{R} \$ 200,00$ $\mathrm{R} \$ 250,00 ; \mathrm{R} \$ 300,00$ e $\mathrm{R} \$ 350,00)$, que totalizam a quantia de $\mathrm{R} \$ 1250,00$, que eqüivale a uma perda líquida de $\mathrm{R} \$$ 250,00 ao final dessas dez cartas. A mesma perda ocorre no baralho "B", pois em uma das dez cartas, o participante perde R \$1250,00 de uma só vez. Assim, o baralho "A" apresenta uma punição mais freqüente, embora com valores menores, enquanto no baralho "B" a punição é menos freqüente, mas de maior valor. Por outro lado, ao virar dez cartas dos baralhos "C" ou "D", os participantes ganham $\mathrm{R} \$ 500,00$ ( $\mathrm{R} \$ 50,00$ a cada carta selecionada), mas o total de suas punições, não previstas, é de apenas $\mathrm{R} \$ 250,00$ acarretando um lucro líquido de R \$250,00. Os baralhos “C” e “D” são também equivalentes em termos de perda líquida. No baralho "C" as punições são mais freqüentes e de menor valor ( $\mathrm{R} \$ 25,00 ; \mathrm{R} \$ 50,00 ; \mathrm{R} \$ 75,00)$, enquanto no baralho “D” a punição é menos freqüente, porém em maior magnitude ( $\mathrm{R} \$ 250,00)$.

Para a adaptação da IGT foram, então, necessários os seguintes procedimentos: (a) tradução simples, na qual foram traduzidos as instruções e os procedimentos da língua inglesa para a língua portuguesa, pela pesquisadora; (b) confirmação da tradução, onde dois juizes com domínio da língua inglesa, pesquisadores da área da neuropsicologia, julgaram a tradução simples como adequada, e uma comparação entre a tradução inicial e a tradução demonstrada em uma publicação em português prévia (A. Damasio, 1996) não observou diferenças constatadas; (c) Adequação sóciocultural, onde com base na análise de dois juizes especialistas independentes, a moeda utilizada no instrumento original (dólar) foi substituída pela moeda brasileira atual (real) e a regra concernente ao valor inicial concedido aos participantes - de U\$2.000,00 para R \$ 0,00. Segundo pressupostos da Teoria Prospectiva (Kahneman \& Tversky, 1979), a configuração da situação de decisão pode influenciar as escolhas, de maneira que situações configuradas em termos de ganho (por exemplo, inicar a tarefa com $\mathrm{R} \$ 2000,00$ ) induziriam a uma conduta conservadora e situações voltadas para perdas levariam à busca pelo risco. Assim, hipotetizou-se que iniciar a tarefa com $\mathrm{R} \$ 0,00$ representaria um valor neutro, e, consequentemente, o indivíduo poderia obter lucro, sem necessariamente adotar uma postura de busca pelo risco; (d) construção do rapport, na qual foi elaborada uma instrução da tarefa, com base nos estudos que utilizaram esse instrumento (Bechara, 2001, 2003; Bechara et al., 1994; Grant, Contoreggi \& London, 2000; 
Manes et al., 2002; Murphy et al., 2001; Rahman, Sahakia, Cardinal, Rogers, \& Robins, 2001; Tranel, Bechara \& Denburg, 2002) e; (e) estudo piloto, no qual realizou-se a aplicação individual da versão adaptada da IGT (Bechara et al., 1994) em uma amostra piloto, onde a partir da avaliação de dois juizes especialistas, sugeriu-se a inclusão de algumas jogadas de treino, para suprir a dificuldade apresentada por aqueles participantes que não tinham contato prévio com computador.

\section{Resultados}

Não houve diferença significativa entre os grupos de diferentes faixas etárias, no que se refere ao número de cartas retiradas de cada um dos baralhos, conforme resultados da Análise de Variância Medidas Repetidas. Conforme mostra a Tabela 2, o número total de cartas selecionadas dos baralhos vantajosos (C,D) e desvantajosos (A,B) não diferiu significativamente entre os grupos $(F(1 ; 80)=$ $0,000 ; p=1,000)$. Também não se verificou um efeito significativo de tipo de baralho $(F(1 ; 80)=0,275 ; p=0,601)$, nem da interação tipo de baralho x grupo $(F(1 ; 80)=0,083$; $p=0,774)$. Considerando os pares de baralhos agrupados pelo critério de frequiência, isto é, conforme a frequiência de punições que o baralho envolve, quais sejam (B e D, baixa freqüência de punições) e (A e C, alta frequiência de punições), não se observou diferença significativa entre os grupos no que se refere ao número de cartas selecionadas dos subgrupos de baralhos (A e C) $(t(65,948)=-0,884 ; p=0,380)$ e $(\mathrm{B}$ e D $)(t(65,948)=$ $0,884 ; p=0,380)$.

Para a avaliação do aprendizado desenvolvido pelos participantes, o desempenho de cada um foi dividido, ao longo de todo jogo em cinco blocos de vinte cartas cada, conforme critério adotado em estudos anteriores de Bechara et al. (1994). Iniciando pelo critério de baralhos vantajosos e desvantajosos, calculou-se o número de seleções dos baralhos C e D (vantajosos) menos o número de cartas selecionadas dos baralhos A e B (desvantajosos) para cada bloco (1, 2, 3, 4 e 5) de vinte cartas. Então, foi derivado um escore final resultante do cálculo de aversão ao risco para cada bloco, a partir da seguinte equação: $(\mathrm{C}+\mathrm{D})-(\mathrm{A}+\mathrm{B})$. Ao aplicar-se um teste de Comparações Pareadas (post hoc Sidak's t-tests), foi possível observar como os blocos (1, 2, 3, 4 e 5) diferiram entre si (Figura 2). O bloco 1 (jogadas de 1 a 20) diferiu significativamente de todos os outros blocos, visto que seu escore médio foi menor que os demais escores. Este dado indica que nesse momento do jogo os participantes selecionaram mais cartas dos baralhos desvantajosos. Até o bloco 4, os dois grupos vão aumentando progressivamente sua preferência aos baralhos vantajosos, mas no último bloco, essa preferência volta a desaparecer. O bloco 4 (jogadas de 61 a 80) difere significativamente de todos os outros blocos, tendo sido seu escore médio maior que os demais escores, indicando uma seleção muito maior de cartas dos baralhos vantajosos em ambos os grupos. Não foi detectada diferença significativa entre os escores obtidos nos blocos 2, 3 e 5 .

O mesmo procedimento conduzido anteriormente, foi utilizado para contemplar o critério de freqüência: baralhos

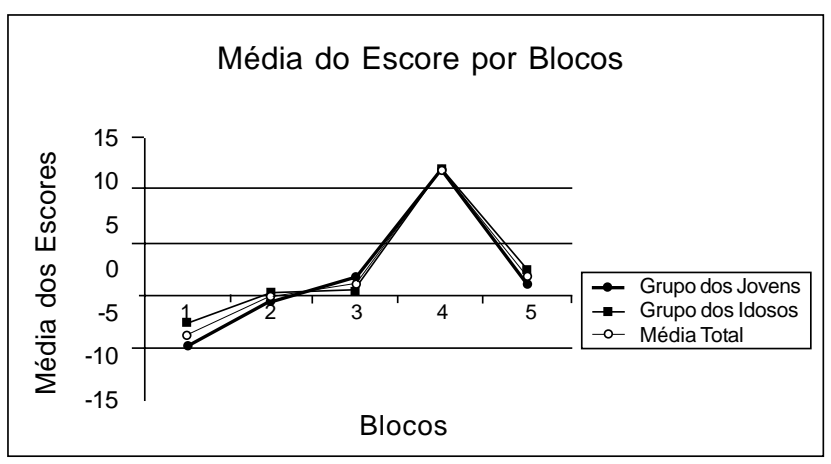

Figura 2. Média dos escores por bloco, segundo critério vantajosos $\mathrm{X}$ desvantajosos, para a amostra total, o grupo de idosos e o grupo de jovens.

Tabela 2

Comparação entre os Grupos em Relação aos Baralhos Desfavoráveis e Favoráveis e entre Baralhos com Maior e Menor Freqüência de Punição

\begin{tabular}{|c|c|c|c|}
\hline Baralhos/grupos & Média & Desvio padrão & N. casos \\
\hline \multicolumn{4}{|c|}{ Baralhos desfavoráveis $a+b$} \\
\hline Jovens & 50,85 & 8,68 & 42 \\
\hline Idosos & 50,25 & 10,38 & 40 \\
\hline \multicolumn{4}{|c|}{ Baralhos favoráveis $\mathrm{c}+\mathrm{d}$} \\
\hline Jovens & 49,14 & 8,68 & 42 \\
\hline Idosos & 49,75 & 10,38 & 40 \\
\hline \multicolumn{4}{|c|}{ Baralhos com maior freqüência de punição $a+c$} \\
\hline Jovens & 4,54 & 43,33 & 42 \\
\hline Idosos & 7,07 & 44,50 & 40 \\
\hline \multicolumn{4}{|c|}{ Baralhos com menor freqüência de punição b+d } \\
\hline Jovens & 4,54 & 56,66 & 42 \\
\hline Idosos & 7,07 & 55,50 & 40 \\
\hline
\end{tabular}


com punição pouco freqüente (B e D) e baralhos com punição frequiente (A e C). Derivou-se um escore final para cada bloco, calculado a partir da seguinte equação: $(\mathrm{B}+\mathrm{D})-$ $(\mathrm{A}+\mathrm{C})$. Análises de Variância Medidas Repetidas, não mostraram efeito de grupo $(F(1 ; 80)=0,798 ; p=0,375)$, efeito principal de bloco $(F(3,602 ; 288,124)=1,728 ; p=0,151)$ nem interação bloco X grupo $(F(3,602 ; 288,124)=0,217$; $p=0,914)($ Figura 3$)$.

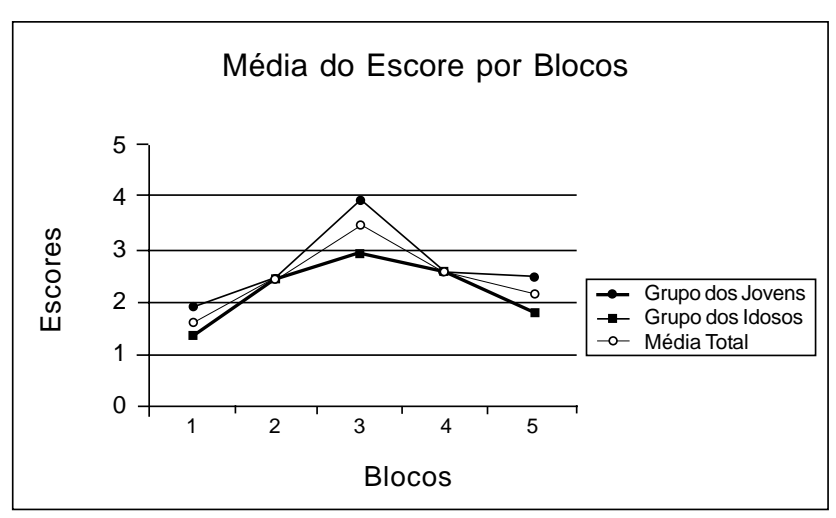

Figura 3. Média dos escores por bloco, segundo critérios de frequência para a amostra total, o grupo de idosos e o grupo de jovens.

\section{Discussão}

Os resultados referentes à ausência de efeito de idade na TD estão em harmonia com alguns dados da literatura mencionados anteriormente (Bechara et al., 1994; Dror et al., 1998; Hershey \& Wilson, 1997; MacPherson et al., 2002; Walker et al., 1997). Uma primeira possibilidade para a ausência de diferenças na TD entre os grupos do presente estudo foi a homogeneidade da amostra quanto às capacidades cognitivas e quanto à ausência dos Transtornos Psiquiátricos avaliados. A pressuposição de que idosos exibiriam decisões diferentes dos jovens, e que até poderiam demonstrar um pior desempenho na TD, é comumente levantada em tarefas que exigem uma grande demanda cognitiva. Não obstante, no presente estudo, apesar das diferenças de idade entre os dois grupos, foram selecionados apenas participantes com bom desempenho cognitivo, sendo mantidos no estudo apenas indivíduos sem maiores déficits de memória.

Além disso, pode-se também postular que áreas corticais subjacentes à TD realmente não sofrem um efeito nítido do envelhecimento normal. Esses achados já foram encontrados em um estudo conduzido por MacPherson et al. (2002), o qual não evidenciou diferenças de idade quanto ao desempenho na maioria das tarefas dependentes da região pré-frontal ventro-mediana, corroborando com a hipótese de que as regiões cerebrais responsáveis pelas funções relacionadas à TD são menos sensíveis aos déficits que naturalmente acompanham o envelhecimento saudável.

Por fim, mas não menos importante, entende-se que por alguma particularidade da aplicação do instrumento no presente estudo, os participantes empregaram, na maior parte das vezes, processos automáticos de assimilação da freqüência da informação, apoiando-se apenas na informação "online". Tal aspecto, além de contribuir para a definição das prefe- rências de escolha diminui a demanda de recursos da memória de trabalho, também contribuindo para a não houvesse disparidade entre os grupos. Neste sentido, o processamento automático das frequêencias de recompensas e punições referentes a cada baralho contribuiu para que se desenvolvesse, inicialmente, uma preferência às recompensas freqüentes (baralhos $\mathrm{B}$ e D) e rejeição às punições freqüentes (baralhos A e C), o que explica a diferença de comportamento da atual amostra, quando em comparação com os estudos de Bechara et al., que têm demonstrado que os participantes saudáveis preferem os baralhos "C" e "D". Os achados em vigor, de preferência aos baralhos "B" e " $D$ ", foram igualmente observados em participantes saudáveis por MacPherson et al (2002) e Wilder, Weinberg e Goldberg (1998).

Algumas teorias explicam que este fato deve-se a uma maior facilidade na representação ou processamento da freqüência de ocorrência do que dos cálculos implicados nesse processo (Hasher \& Zacks, 1984). Isto é, quando os indivíduos deparam-se com informações ambíguas e precisam tomar decisões, eles confiam na codificação de informação de frequiências, mais do que na soma de recompensa. Essa aquisição de informação tem sido chamada processo de armazenamento automático (Hasher \& Zacks, 1979, 1984).

Já à luz da teoria que embasa os estudos de Bechara, a Hipótese do Marcador Somático (A. Damasio, 1996, 2003), o desenvolvimento de uma preferência ocorre de forma implícita. Isto é, a partir de um processo de associação entre fatos ou eventos específicos e determinadas sensações somáticas, o indivíduo representa certas situações como seguras ou ameaçadoras. Dessa forma, o processo de associação que sucede ao longo da IGT, com base nesse construto teórico, se dá através da conexão entre categorias conceituais específicas e seus sistemas emocionais relacionados. $\mathrm{Na}$ medida em que o indivíduo vai interagindo com o instrumento, ele gradualmente categoriza as situações experienciadas, sua estrutura, seus componentes e seu significado em termos de sua narrativa pessoal. Assim, são formadas categorias conceituais (mentalmente e no nível neural associado), as quais são conectadas com os sistemas cerebrais usados para a disparada de emoções.

$\mathrm{O}$ aprendizado estímulo-reforçamento, que ocorre implicitamente ao longo das jogadas, parece ser um fator essencial para o desenvolvimento do marcador somático, conduzindo às preferências. Similarmente, faz-se necessária certa flexibilidade para que o aprendizado reverso se consolide, na medida em que as contingências de reforçamento são modificadas (Bechara et al., 1994; Clark, Cools \& Robbins, 2004; Fellows \& Farah, 2003a, 2003b). A memória de trabalho, principalmente seu componente executivo que manipula as informações armazenadas, também tem sido evidenciada como um fator essencial na formação das categorias associativas que levam à decisão (Bechara, Damasio \& Damasio, 2000a; Bechara et al., 1998; Bechara \& Martin, 2004; Martin et al., 2004).

A partir disso, o comportamento da amostra em questão pode ser entendido com base em uma confluência destes dois aportes teóricos: hipótese do marcador somático e teorias 
sobre o processo de armazenamento automático. Na atual amostra, na maior parte da tarefa, os participantes associaram os baralhos com maior frequêencia de punição como ameaçadores e aqueles com menor frequência como seguros. Isto porque, ao formar as primeiras categorias conceituais concernentes ao instrumento, em virtude de um processamento cognitivo automático referente à freqüência de ocorrência, eles detiveram-se apenas na característica de freqüência de punições e recompensas dos baralhos, sem levar em consideração a magnitude desses ganhos ou perdas. A partir de então, toda jogada que, apesar de incluir recompensas também contou com punições, foi conceituada como desvantajosa, não sendo levado em conta o valor dessa perda. Dessa forma, uma emoção positiva, ou menos negativa, foi associada com àqueles baralhos cuja frequêencia de punição era menor.

Ao considerarem-se as duas curvas de aprendizado conjuntamente, é possível visualizar que, no momento em que há uma diminuição nas escolhas pelos baralhos "B" e "D" (quarto bloco do critério frequêencia), há um aumento no número de seleções de cartas dos baralhos "C" e "D" (quarto bloco do critério vantajosos e desvantajosos), o que pode ser entendido por um abandono gradual dos processos automáticos com relação aos intencionais, que culminou nesse momento do jogo. É plausível pensar que, enquanto processavam automaticamente a frequiência de ocorrência, dando preferência, dessa forma, aos estímulos positivos mais freqüentes, os indivíduos realizavam paralelamente um processo de aprendizagem da tarefa, com relação às quantias de valores ligadas a cada baralho. No quarto bloco (critério vantajosos e desvantajosos), esse processamento paralelo culminou em um entendimento mais elaborado e consciente acerca da tarefa, interferindo no processamento automático da frequiência. Essa mudança de comportamento permitiu aos participantes uma conceitualização mais clara e apurada da tarefa. Porém, surpreendentemente, no quinto bloco de ambos os agrupamentos, os indivíduos não mantêm a preferência por nenhum dos padrões de escolha, desenvolvidos anteriormente.

Visto que o declínio no quinto bloco ocorreu em ambos os agrupamentos, é possível que o aprendizado simultâneo de ambos padrões (frequiência e magnitude) durante toda a tarefa, não tenha permitido uma consolidação forte o suficiente de cada um deles, capaz de manter-se estável até o final. Outra alternativa é uma queda na motivação dos participantes, haja vista a complexidade de fatores envolvidos na tarefa, que exige uma atenção contínua a diversas características associadas a cada baralho (valor, probabilidade, consequências imediatas e em longo prazo). No entanto, como nenhuma destas alternativas foi diretamente avaliada, tratam-se apenas de conjecturas teóricas, que podem ser verificadas em futuros experimentos.

O resultado do presente estudo, que demonstra desempenhos similares entre grupos de diferentes faixas etárias, parece fortalecer a hipótese de que o envelhecimento cognitivo, apesar de ocorrer principalmente na região pré-frontal (West, 1996), apresenta um declínio dissociado através das distintas estruturas associadas ao córtex pré-frontal (Denburg, Tranel \& Bechara, 2005; Lamar \& Resnick, 2004; MacPherson et al., 2002). Neste sentido, alguns estudos referem a região dorsolateral, supostamente menos envolvida no desepenho dos indivíduos na IGT, como primeiramente afetada pelo envelhecimento (West, 1996). A faixa etária do grupo de idosos da presente amostra também pode ser um fator contribuinte para a ausência de diferenças, visto que idosos com idade mais elevada poderiam apresentar maiores comprometimentos cognitivos.

Outro fator a ser considerado diz respeito às características específicas da amostra que, em vista do estudo de um fator específico, impedem a generalização dos resultados para todos idosos: (a) todos os indivíduos não apresentavam maiores comprometimentos de memória, conforme mensurados a partir de seu desempenho no MEEM e na escala critérios de positividade para déficit cognitivo e (b) os participantes idosos são, em sua maioria, pertencentes a uma classe social privilegiada e possuidores de um nível alto de instrução, o que os diferencia da população de idosos como um todo.

Cabe ainda observar que aspectos específicos do processo de adaptação da IGT podem ter exercido alguma influência nos resultados contemplados na presente amostra. Neste sentido, ressalta-se a alteração concernente ao valor inicial concedido aos participantes. Com base no comportamento evidenciado por ambos os grupos, pode-se hipotetizar que iniciar a tarefa sem dinheiro contribuiu para um padrão de escolhas conservador, visto que as opções iniciais envolveram baralhos com baixa frequiência de punições. Contudo, também é plausível considerar que um valor base de $\mathrm{R} \$ 2000,00$ poderia favorecer, por exemplo, uma precoce rejeição do baralho "B", que envolve punições de grande magnitude, embora ocasionais.

Tendo em vista que este é o primeiro trabalho visando adaptar a IGT para a população brasileira, diferenças entre os achados do presente estudo e os dos que elaboraram o instrumento podem estar no rapport construído com base nos estudo da equipe norte americana ou no valor que os participantes recebem ao iniciar a tarefa. Tendo em mente essas questões, sugere-se a continuidade do processo de adaptação conduzido, seguida de uma nova aplicação do instrumento, para verificar se há algum efeito de instrução deste nas escolhas realizadas pelos indivíduos.

\section{Referências}

Amorim, P. (2000). Mini International neuropsychiatric interview (M.I.N.I): Validação de entrevista breve para diagnóstico de transtornos mentais. Revista Brasileira de Psiquiatria, 3, 106-115.

Ballesteros Jiminez, S., \& Garcia Rodríguez, B. (1996). Procesos psicológicos básicos. Madrid, España: Universitas.

Bechara, A. (2001). Neurobiology of decision-making: Risk and reward. Seminars in Clinical Neuropsychiatry, 6(3), 205-216. 
Bechara, A. (2003). Risky business: Emotion, decision-making, and addiction. Journal of Gambling Studies, 19(1), 23-51.

Bechara, A., Damasio, A. R., Damasio, H., \& Anderson, S. (1994). Insensitivity to future consequences following damage to human prefrontal cortex. Cognition, 50, 7-15.

Bechara, A., Damasio, H., \& Damasio, A. R. (2000a). Emotion, decision-making, and the orbitofrontal cortex. Cerebral Cortex, 10, 295-307

Bechara, A., Damasio, H., Tranel, D., \& Anderson, C. (1998). Dissociation of working memory from decision making within the human prefrontal cortex. The journal of neuroscience, 18(1), 428-437.

Bechara, A., Damasio, H., Tranel, D., \& Damasio, A. (1997). Deciding advantageously before knowing the advantageous strategy. Science, 275, 1293-1295.

Bechara, A., \& Martin, E. M. (2004). Impaired decision making related to working memory deficits in individuals with substance addictions. Neuropsychology, 18(1), 152-162.

Bechara, A., Tranel, D., \& Damasio, A. (2000b). Poor judgment in spite of high intellect. In R. Bar-On \& J. Parker (Eds.), The handbook of emotional intelligence (pp. 193-215). San Francisco: Jossey-Bass.

Bechara, A., Tranel, D., Damasio, H., \& Damasio, A. R. (1996). Failure to respond autonomically to anticipated future outcomes following damage to prefrontal cortex. Cerebral Cortex, 6, 215-225.

Chaves, M. L., \& Izquierdo, Y. (1992). Differential diagnosis between dementia and depression: A study of efficiency increment. Acta Neurologica Scandinavia, 85, 378-382.

Clark, L., Cools, R., \& Robbins, T. W. (2004). The neuropsychology of ventral prefrontal cortex: Decision-making and reversal learning. Brain and Cognition, 55, 41-53.

Damasio, A. (1996). O erro de Descartes: Emoção, razão e cérebro humano. São Paulo, SP: Companhia das Letras.

Damasio, A. (2003). Looking for Spinoza: Joy, sorrow and the feeling brain. London: William Heinemann.

Damasio, H. (1996). Neurobiology of decision-making. In A. R. Damasio, H. Damasio \& Y. Christen (Eds.), Neurobiology of decision-making (pp. 1-12). Berlim, Germany: Springer.

Denburg, N., Tranel, D., \& Bechara, A. (2005). The ability to decide advantageously declines prematurely in some normal older persons. Neuropsychologia, 43(7), 1099-1106.

Dror, I. E., Katona, M., \& Mungur, K. (1998). Age differences in decision making: To take a risk or not? Gerontology, 44(2), 67-71.

Eysenck, M. W., \& Keane, M. T. (1994). Psicologia cognitiva: Um manual introdutório. Porto Alegre, RS: Artes Médicas.

Fellows, L. K., \& Farah, M. (2003a). Different underlying impairments in decision-making following ventromedial and dorsolateral frontal lobe damage in humans. Cerebral Cortex, 15, 58-63.

Fellows, L. K., \& Farah, M. (2003b). Ventromedial frontal cortex mediates affective shifting in humans: Evidence from a reversal learning paradigm. Brain, 126, 1830-1837.
Ferreira, E. D. (1997). Avaliação de pacientes com demência do tipo Alzheimer e multinfarto: Um estudo de coorte. Dissertação de Mestrado não-publicada, Universidade Federal do Rio Grande do Sul, Porto Alegre, RS.

Finuncane, M. L., Alhakami, A., Slovic, P., Hibbard, J. H., Peters, E., Mertz, C. K., \& MacGregor, D. G. (2002). Aging and decision making competence: An analysis of comprehension and consistency skills in older versus younger adults considering health-plan options. Journal of Behavioral Decision Making, 15(2), 141-167.

Folstein. M. F., Robins, L. N., \& Helzer, J. E. (1983). The MiniMental State Examination. Archives of General Psychiatry, 40, 812.

Grant, S., Contoreggi, C., \& London, E. (2000). Drug abusers show impaired performance in a laboratory test of decision making. Neuropsychologia, 38, 1180-1187.

Hasher, L., \& Zacks, R. T. (1979). Automatic and effortful processes in memory. Journal of Experimental Psychology: General, 108, 356-388.

Hasher, L., \& Zacks, R. (1984). Automatic processing of fundamental information: The case of frequency of occurrence. American Psychological Association, 39(12), 1372-1388.

Hershey, D. A., \& Wilson, J. A. (1997). Age differences in performance awareness on a complex financial decision making task. Experimental Aging Research, 23, 257-273.

Kahneman, D., \& Smith, V. (2002). Foundations of behavioral and experimental economics: Daniel Kahneman and Vernon Smith. The prize in economic sciences. Stockholm, Sweden.

Kahneman, D., \& Tversky, A. (1979). Prospect theory: An analysis of decision under risk. Econometrica, 4, 263-291.

Lamar, M., \& Resnick. (2004). Aging and prefrontal functions: dissociating orbitofrontal and dorsolateral abilities. Neurobiology of Aging, 25, 553-558.

MacPherson, S. E., Phillips, L. H., \& Sala, S. D. (2002). Age, executive function, and social decision making: A dorsolateral prefrontal theory of cognitive aging. Psychology and Aging, $17(4), 598-609$.

Manes, F., Shakian, B., Clark, L., Rogers, R., Antoun, N., Aitken, M., \& Robbins, T. (2002). Decision-making process following damage to the prefrontal cortex. Brain, 125, 624-639.

Martin, E. M., Pitrak, D. L., Weddington, W., Rains, N. A., Nunnally, G., Nixon, H., Grbesic, S., Vassileva, J., \& Bechara, A. (2004). Cognitive impulsivity and HIV serostatus in substance dependent males. Journal of the International Neuropsychological Society, 10(7), 931-938.

Medin, D. L., \& Ross, B. H. (1992). Cognitive Psychology. San Diego, CA: Harcourt Brace Jovanovich College.

Mellers, B. A., Schwartz, A., \& Cooke, A. D. J. (1998). Judgment and decision making. Annual Review of Psychology, 50, 447-477.

Murphy, F. C., Rubinsztein, J. S., Michael, A., Rogers, R. D., Robbins, T. W., Paykel, E. S., \& Sahakian, B. J. (2001). Decisionmaking cognition in mania and depression. Psychological Medicine, 31(4), 679-693.

Palmini, A. (2004). O cérebro e a tomada de decisões. In P. Knapp (Ed.), Terapia cognitivo-comportamental na prática psiquiátrica (pp. 71-88). Porto Alegre, RS: Artes Médicas.

Petersen, R., Smith, G., Waring, S., Ivnik, R., Kokmen, E., \& Tangelos, E. (1997). Aging, memory and mild cognitive impairment. International Psychogeriatric, 9, 65-69. 
Plous, S. (1993). The Psychology of judgment and decision making. New York: McGraw- Hill.

Rahman, S., Sahakia, B. J., Cardinal, R. N., Rogers, R. D., \& Robins. T. W. (2001). Decision making and neuropsychiatry. Trends in Cognitive Sciences, 5(6), 271-277.

Sanfey, A. G., \& Hastie, R. (2001). Judgment and decision making across the adult life span: A tutorial review of psychological research. In D. Park \& N. Schwarz (Eds.), Aging and cognition (pp. 253-273). Philadelphia, PN: Psychology Press.

Sheehan, D. V., Lecrubier, Y., Sheehan, K. H., Amorim, P., Janavs, J., Weiller, E., \& Hergueta, T. et al. (1998). The Mini International Neuropsychiatry Interview (M.I.N.I.): The development and validation of a structured diagnostic psychiatric interview for DSM-IV and CID-10. Journal of Clinical Psychiatry, 59(20), 22-33.

Tranel, D., Bechara, A., \& Denburg, N. (2002). Asymmetric functional roles o right and left ventromedial prefrontal cortices in social conduct, decision-making, and emotional processing. Cortex, 38, 589-612.
Tversky, A., \& Kahneman, D. (1974). Judgment under uncertainty: Heuristics and biases. Science, 185, 1124-1131.

Walker, N., Fain, W. B., Fisk, A. D., \& McGuire, C. L. (1997). Aging and decision making: Driving-related problem solving. Human Factors, 39(3), 438-444.

West, R. L. (1996). An application of prefrontal cortex function theory to cognitive aging. Psychological Bulletin, 120, 27 1-292.

Wilder, K. E., Weinberger, D. R., \& Goldberg, T. E. (1998). Operant conditioning and the orbitofrontal cortex in schizophrenic patients: Unexpected evidence for intact functioning. Schizophrenia Research, 30, 169-174. 